\title{
Communication
}

\section{Geometrical transformations and development of geometry}

\author{
Joaquin Giménez $^{1 *}$, Xhevdet Thaqi ${ }^{2}$ and Yuly Vanegas ${ }^{3}$ \\ 1 Universitat de Barcelona; quimgimenez@ub.edu \\ 2 Public University "Kadri Zeka"; xhevdet.thaqi@uni-gilan.net \\ 3 Universitat de Lleida; yuly.vanegas@udl.cat \\ *Correspondence: xhevdet.thaqi@uni-gjilan.net
}

\begin{abstract}
A way of systematizing different geometries by geometrical transformations is presented by using Klein's perspective. Symmetry is considered the basic precedent, and different groups of transformations are introduced by using Erlangen program ideas. So, to focus on the importance of geometric transformations, two purposes are shown: first, the classification of geometries is based on the invariants of geometric transformations; and second, the construction of geometry unlike the theory of deduction can be done by means of geometric transformations.
\end{abstract}

Keywords: geometry, geometrical transformations; symmetry; invariance, perspectivity, Möbius transformations.

\section{Introduction}

Symmetry and perspective have been permanent motivations and objectives in research, and not only are they logical and philosophical principles, but these notions have captivated philosophers, painters, and poets for many years. Our intention is to reflect on their meaning in order to show how it is possible to use these principles of symmetry and perspective to better understand multiple scientific theories and easily obtain concrete results.

Symmetry. We chose Weyl's explanation, which uses the word "symmetry" in two somewhat different senses: something proportioned and harmonious that has certain geometric regularities, or a certain process of repetition [1].

How can we define precisely what we understand by symmetry? To define it, we can at least ask ourselves about the nature of this concept and try to answer some questions: What is symmetry? Is it a property, or is it a principle? If it is the latter, is it an experimental law, a postulate, a rule of induction, an aesthetic imperative, or something else? In fact, we believe that it can be each of these things, depending on the circumstances, and it is to this question that we will devote our attention.

The first manifestation of the use of symmetry is found in Archimedes of Syracuse's (287-212 BC) theory of balance [2]. His first postulate is: Equal weights at equal distances are kept in balance. Archimedes can be considered the "father" of mathematical physics, as defender and exponent of the so-called not pure mathematics but applied to nature or other artifacts. It is almost certain that he should have stated his postulate based on reasons of symmetry.

The Pythagorean school already highlighted symmetry and/or harmony as a method of achieving beauty: What is the wisest thing? The number. What is the most beautiful? Harmony. Thus, began the study of symmetries and harmonies, both in mathematics and in other sciences.

We can recall the preference that the Archimedean tradition has given to theoretical arguments over experimental justification, when possible. This preference, which is still maintained among mathematical physicists, is partially due to the fact that one can imagine idealized situations that cannot be performed experimentally but are very valuable 
aids in the construction of theories. Modern physics, even when dealing with pure abstract principles, is very useful to carry out mathematical reasoning.

The basic algebraic structure in the study of symmetries is the group. The concept of group appears for the first time in the context of groups of permutations of the roots of an algebraic equation. The important point to note is that in the very genesis of the group concept, it appears as a set of transformations that leave something invariant. More explicitly, if $S$ is an arbitrary set, we can call a bijection of $S$ into itself a transformation of $S$, so that each point has an image and is the image of a single point.

We can quickly think of examples and see how this definition generalizes our idea of symmetry. For example, $S$ can be a plane and $T$ (invariant) the set of points of a figure in that plane. Let $\mathrm{P}$ be a point on the plane and $\mathrm{s}$ and $\mathrm{t}$ two non-parallel lines in the same plane. A rotation is the composition of two reflections on these lines, as follows: First, point $\mathrm{P}$ is reflected on straight line $\mathrm{s}$, and then its image is reflected with respect to line $t$. The point of intersection of the two lines is known as the center of rotation. The transformation of rotation $\Phi$ in the plane would be a symmetrical transformation when $\mathrm{T}$ is considered to be any one of a whole family of concentric circles, but it would not be if $\mathrm{T}$ is a line of the plane. Let $\mathrm{P}$ be a point in a plane and $\mathrm{s}$ and $\mathrm{t}$ two parallel straight lines in the same plane. A translation is the composition of two reflections on these lines, as follows: Point $\mathrm{P}$ is first reflected in line $\mathrm{s}$, and then this reflection is reflected in line $\mathrm{t}$. A translation in the plane would be a symmetry for each line that has the direction of the translation vector, but not for other lines, whose images would be parallel lines, but not coincident with it. When the transformation considered is a reflection on a vertical line, the symmetrical figures, in the sense that we have just said, are precisely those that "have bilateral symmetry" in the most colloquial language.

The important thing is that this concept admits an immediate generalization. Thus, if in $S$ we do not have a privileged subset $T$, but rather $S$ is endowed with some structure or property, we can say that a transformation of $S$ is a symmetry of the structure, or even a symmetry of $S$, when in this it is implicitly assumes its structure if $\Phi$ preserves that structure. For example, if a metric space structure is defined in $S$, that is, a "distance between two points" function $d$, then $\Phi$ will be a symmetry when $d(\Phi(x), \Phi(y))=d(x, y)$, with $x, y \in S$. This is the case of rotations and translations in the plane, but not the case of homothecies, for which $\mathrm{d}(\Phi \lambda(\mathrm{x}), \Phi \lambda(\mathrm{y}))=|\lambda| 2 \mathrm{~d}(\mathrm{x}, \mathrm{y})$ when $\Phi \lambda(\mathrm{x})=\lambda \mathrm{x}$, and $\lambda \in \mathrm{R}$.

As another example, if $S$ were endowed with a vector space structure, then the symmetries of $S$ would be the isomorphisms of the vector space $S$, for which the linear structure is preserved, $\Phi(\mathrm{x}+\mathrm{y})=\Phi(\mathrm{x})+\Phi(\mathrm{y}), \Phi(\lambda \mathrm{x})=\lambda \Phi(\mathrm{x})$. In fact, a transformation of $\mathrm{S}$ induces a symmetry of the vector space of the real functions defined in $S$ by $(U F)(x)=F(\Phi$ $-1(x)$ ). If $S$ is endowed with a topological structure, the symmetries of $S$ will be precisely the "homeomorphisms", that is, continuous invertible transformations whose inverse is also continuous. If $S$ is a real vector space endowed with an internal product structure, the symmetries of $S$ will be orthogonal transformations, and so on.

Let us also indicate that an abstract group can be realized as a group of transformations of spaces $\mathrm{M}$. Therefore, we understand that there is a correspondence that associates with each element $g \in G$ a transformation $\Phi g$ of $M$ so that the neutral element $\mathrm{e}$ corresponds to the identity in $\mathrm{M}, \Phi \mathrm{e}=\mathrm{idM}$, and for each pair of elements in $\mathrm{G}, \Phi \mathrm{g} 1 \circ \Phi \mathrm{g} 2$ $=\Phi g 1 g 2$. This action of $\mathrm{G}$ in $\mathrm{M}$ allows us to introduce an equivalence relation in $\mathrm{M}$. In the case that $M$ is endowed with some structure, the interesting realizations of $G$ will be those in which $\Phi g$ is a symmetry, for each $g \in G$.

The importance of the use of the concept of symmetry in mathematics has been increasing fundamentally since the last quarter of the 19th century, when the concept of geometry itself was related to the study of invariants under groups and with great utility manifested by Lie groups of symmetries of differential equations in their resolution. However, the definitive success of group theory in mathematics was obtained around 1875, when, thanks to the work of Klein and Lie, the "unification of geometries" was achieved, establishing "the group of transformations as the guiding principle of any type of geometry". Before Klein and Lie's work, a lot of work had been done by some well-known 
mathematicians, whom we will briefly talk about in the following. For further study of isometric transformations, we recommend the book Indra's Pearls: The Vision of Felix Klein by David Mumford, Caroline Series, and David Wright [3].

Perspectivity - expanded and different vision of parallelism and perpendicularity. The French mathematician Gérard Desargues (1591-1661), in his study of conics, introduced a mathematical foundation for perspective theory (two-dimensional representation of three-dimensional objects). His development of the fundamental projective notions and new methods of demonstration (now valid for all conics, unlike the Greeks, who had different methods for different cases) represent significant advances since the time of Apollonius. Influenced by him, the developments were continued by Blaise Pascal (1623-1662) and Philippe de Hire (1649-1718).

We will return in this paper to some of the fundamental themes worked on the re-al projective plane, knowing that the theory is much more general. We believe that it is one of the topics that should be known, because collineations, and with them projective invariants, appear in a context that allows them to be easily distinguished from trans-formations.

We consider the geometric properties arising from perspective problems, for ex-ample, of an image captured (projected) by a painter from the original on a canvas, where the center is considered the artist's eye. In this process, lengths and angles are distorted in a way that has to do with the relative positions of the objects. Still, the geometric structure of the original is recognizable on the canvas. This is due to the existence of certain projective invariants, that is, properties that appear "unchanged" on the canvas and make identification possible.

Clearly, these invariants are not lengths, angles, or congruences. Finding and analyzing these invariants is the object of projective geometry. It should be noted that a systematic study in this regard was only carried out in the late 18th century, when a French officer, J.V. Poncelet (1788-1867), wrote his famous Traité des propriétés projectives des figures in 1813.

A "realistic" drawing of an everyday scene is achieved by projecting from a "focus" (hypothetical eye) onto the canvas $\Pi$. That is, each point $x$ in the scene corresponds to a point on the canvas $\Pi$, taking the line that passes through the focus and $x$ and intersecting it with $\Pi$.

The best illustration is the case of a railway (Figure 1): We call the two vertices on one horizontal side of the tile 0 and 1 , and the corresponding vertices on the opposite side $0^{\prime}$ and $1^{\prime}$, so that the diagonal passes through $0^{\prime}$ and 1 . Then the line from $1^{\prime}$ to $\infty \mathrm{d}$ is just where the diagonal of the next tile is drawn, whose horizontal intersection with 0 and 1 gives us vertex 2 , corresponding to the following tile. Now, drawing the vertical by 2 , its intersection with the other rail gives us vertex 2 ', thus completing the data to draw tile 1 (we start with 0 ). 


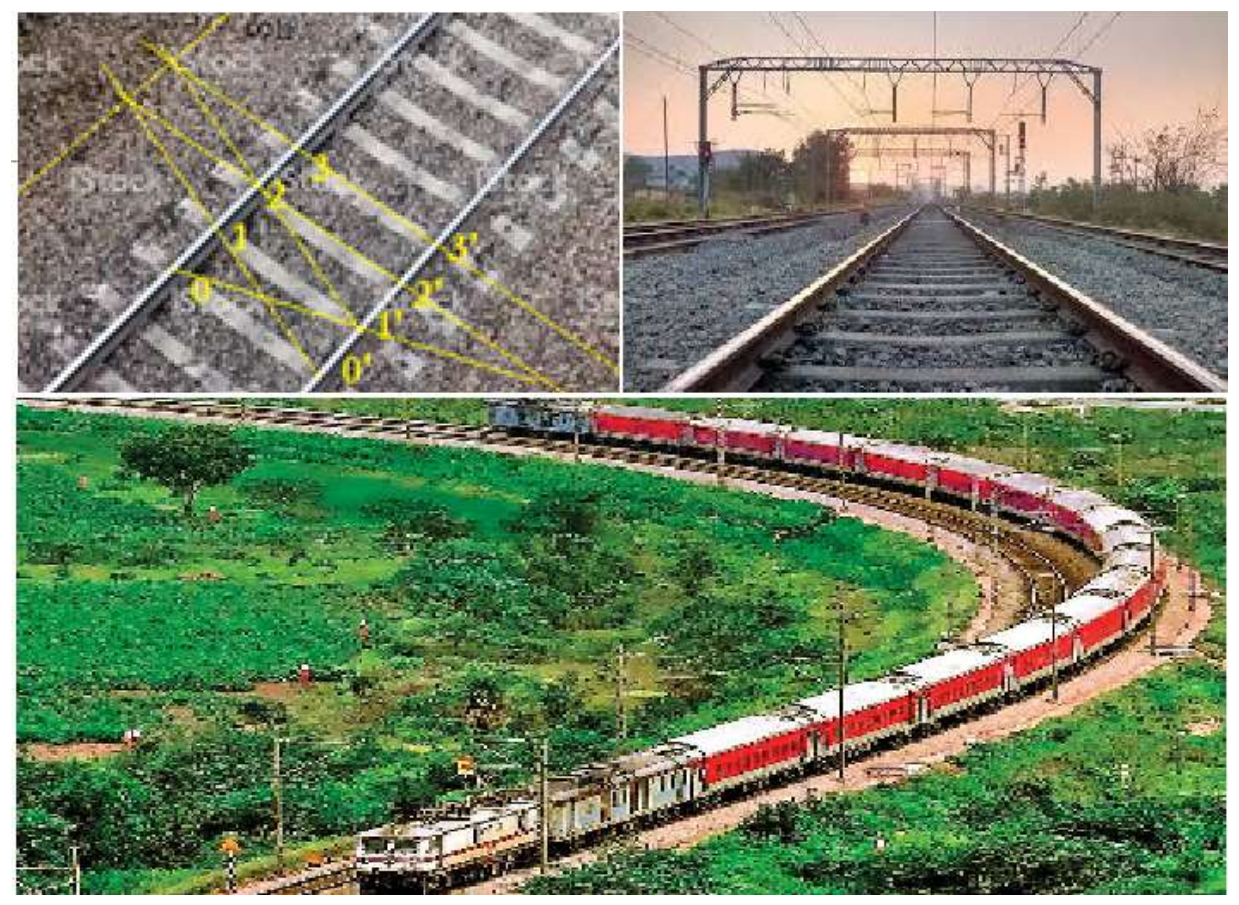

Figure 1. Geometric properties arising from perspective problems.

This process can be iterated to find the sleepers of the horizontal railway that we want. This method has been known since the Renaissance. Note that we do not even need a compass anymore; it works with pure rule. And it works well, which indicates that it must support theorems. For example, why is it that the intersection points of the diagonals with the verticals $0^{\prime}$ and 1 with $2^{\prime}$ and $2,1^{\prime}$ and 2 with $3^{\prime}$ and $3,2^{\prime}$ and 3 with $4^{\prime}$ and 4 , etc., are all aligned with $\infty \mathrm{h}$ ? Since the Renaissance it is known from experience that this is the case, but the mathematical foundations took centuries to develop. For the moment, let us leave this as motivation to study and explore the meaning and definition of invariant.

More generally, we can find a state-of-the-art approach in Zisserman and Mundy [4] for both mathematical aspects and applications. These applications concern difficult basic problems of vision and seem to be a new useful way to solve them. As invariants are numerical quantities associated with topological features, they can be used to index models or images to recognize objects represented in images [5].

\section{Theoretical background - Consideration on invariants}

There are two major fields of work within the new considerations on invariants. First is a precise study of the invariants by clarifying their theory, robustness, and numerical stability. The computation of new invariants associated with curves or 3D configurations of points, lines, and conics is also important [2]. Second is the practical use of these invariants to solve vision problems, an interest clearly shown by Kapur and Mundy [6]. Given a geometric configuration $\alpha$ of points, lines, and curves, an invariant is a numerical quantity $f(\alpha)$ from this configuration that remains the same when the configuration is transformed by a geometric function. If $\Psi$ is such a function, the invariant function should verify $f(\alpha)=f(\Psi(\alpha))$. Given another configuration $\beta$, it is potentially the same as $\alpha$ if $f(\beta)=$ $f(\alpha)$, and this is a necessary condition. If the configuration is composed by sub-configurations $\alpha=\left(\alpha_{1}, \alpha_{2}, \ldots, \alpha_{\mathrm{n}}\right)$, an invariant function can be used as prediction function. If one knows $\alpha_{1}, \alpha_{2}, \ldots, \alpha_{\mathrm{n}-1}$ and $\mathrm{f}(\alpha)$, the value of $\alpha_{\mathrm{n}}$ can be computed if $f$ is invertible.

The nature of the invariants that exist in a problem mainly depends on the geometric function set. Angles and length ratios are the basic invariants associated with similarities; the length ratios of collinear points play the same role with affine transformations, as do 
the cross ratios of collinear points or the concurrent lines with collineations. The main problem is to find the invariant functions that exist in concrete problems. It is known that $f$ is an invariant function, and $g \circ f$ is another one for any real function $g$. The question is how many independent invariants exist for a given problem and how to find them.

We note the interpretation of the general formulation by Gross and Quan [7]. Let E be a set called the space of configurations and let $G$ be a set of transformations on that space $E$ : the elements of $G$ are functions from $E$ into $E$. Consider a particular configuration $x \in E$, to which we apply different transformations of $\mathrm{G}$ (Figure 2).

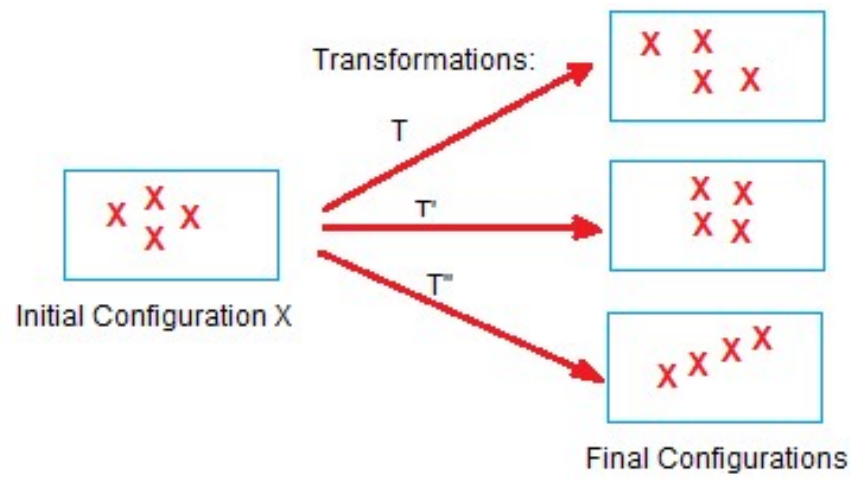

Figure 2. Invariant meaning.

We would like to answer these questions:

- Can we reach all the configurations of $\mathrm{E}$ from the configuration $\mathrm{x}$ ?

- If not, can we characterize the set of configurations reachable from a given configuration?

- If such a characterization is possible, how many independent parameters are needed?

These parameters are called invariants for the set of transformations $\mathrm{G}$. They may be expressed as functions from the configuration space into the set of real numbers R. We can consider the case of differential invariants, i.e., when derivatives of curves or surfaces are involved. Such a case may lead to an infinity of invariants, as the derivatives of a curve can be taken an infinite number of times or at an infinite number of points.

As we would like to analytically calculate the invariants of a given problem, we make a few hypotheses. The first is that set $\mathrm{G}$ is a group acting on E. Elements of $\mathrm{G}$ are functions from $E$ into itself, and $G$ can be provided with the function composition operation, which will be denoted either multiplicatively or by the symbol o, and the axioms which define a group can be proved.

Intuitively, a group acting on set $\mathrm{E}$ is a set of functions from $\mathrm{E}$ to $\mathrm{E}$ which has the three basic properties of all groups: the set contains the identity function, and every function of the set is invertible. For example, a set of rotations around the origin is a group and acts on any affine plane. Since we want to calculate invariants analytically, we want to have real parameters on the configuration set. Just for illustration, we can mention examples of invariants [7]:

- $\quad$ For the group of planar rotations whose center is the origin, the angle is a curvilinear parameter.

- The planar displacement can decompose into a rotation around the origin followed by a translation; the rotation has its angle as a parameter, and the translation has the coordinates of the translation vector as parameters.

- The 3D displacement group can decompose into 3D rotation followed by translation along the axis of rotation, thus the axis direction, axis position, rotational angle, and translation vector are parameters. 
- For transformations of the plane figure in another plane figure conserving perimeter (i.e., squares are transformed to quadrilaterals), the perimeter is the invariant, and we call these isoperimetric transformations.

- For similarity transformations, perpendicular lines are transformed into perpendicular lines, parallel lines are transformed into parallel lines, circle are transformed into circles; i.e., parallelism and perpendicularity are invariant by a similarity transformation.

- For rigid metric transformations, the distance between two points and the magnitude of the angle between two lines are invariants.

- For transformations of a plane figure in another plane figure conserving superficies (i.e., tangram transformed to a determined figure), the superficies is the invariant, and we call these equivalent transformations [8].

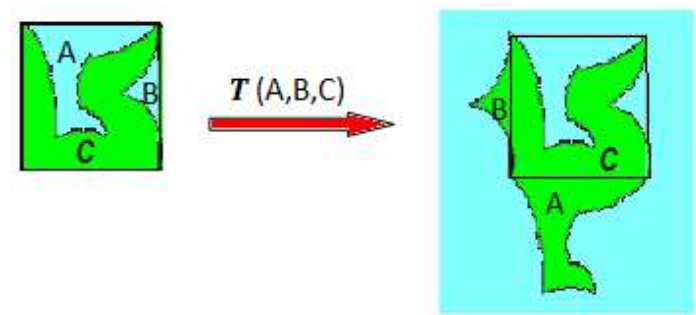

Figure 3. Example of transformation activity.

Now we are dealing with developments in the building and classification of geometries based on groups of projective transformations.

\section{Development of geometry through transformations}

If we see geometric concepts such as "plane", "line", etc., as concrete (physical) or ideal (abstract) "things", in principle we are referring to Plato [6]. From this perspective, a demonstration of the truth of the propositions of mathematical theories is understood as a logical demonstration from the axioms. This organization of mathematics, in Greek mathematics, introduces a novel element: the deductive method, as a validation criterion embodied in the elements of Euclid. These are the elements that make up a demonstrative science: the definitions, postulates, and axioms, and finally the deductive body. From the postulates of the Euclidean system, the first four translate obvious properties, but the fifth is striking for its greater complexity and the lack of intuitive evidence that the others have.

The crisis of foundations occurred in mathematics in the 19th century, when Frege first attempted to solve it by means of a logistics program [9] he initiated to provide arithmetic with certain foundations. Another attempt to overcome the fundamental crisis was the formalist program initiated by Hilbert. For Hilbert, there are no mathematical objects (unlike Platonism), there are only ostensive symbols. The first objective of the formalist program is the "complete formalization" of a deductive system. Geometry underwent radical changes through Hilbert's Foundations of Geometry.

In Hilbert's program, the truth character of the axioms is not considered; the fundamental thing is that it is consistent. For example, there should be, in addition to the uniqueness axiom of the parallel by a point outside a line, another axiom that affirms the existence of more than one parallel through a point outside a line (Lobachevski's axiom), or states that there is no parallel by a point outside a line (Riemann axiom). Hilbert elaborates the process of geometric structuralizing in absolute geometry based on all axioms, except axioms of parallelism. To maintain the consistency and non-contradiction of the axioms, Lobachevski's axiom is added to absolute geometry, and the new deductive system is called hyperbolic geometry; if we add Playfair's axiom (equivalent to Euclid's fifth postulate) to absolute geometry, the deductive system refers to Euclidean geometry, and if we add Riemann's axiom, it refers to elliptical geometry.

From this formalist point of view, the question of the truth or falsity of a geometric statement is meaningless in the axiomatic state. What we can ask is meaningful for the 
consistency, the (non)contradiction, and the (in)completeness of the system. According to Hilbert, the three systems - Euclidean, hyperbolic, and elliptical geometry-are systems of deductive statements that meet these three principles.

Contemporary formalism is a descendant of Hilbertian formalism but is not the same. In the elaboration of the new programs, an attempt was made to achieve internal coherence from the point of view of the geometric contents specified in the systematic and coherent development of geometry through the concept of transformation [9].

The development of geometry through transformations refers to Klein's Erlangen program. Klein combines the development achieved by non-Euclidean and projective geometries with the theories of invariants and groups of transformations. He presents in his program, through groups and subgroups, a systematization and hierarchization of all geometries, conceiving as the object of each geometry the discovery of invariant properties with respect to a certain group of transformations. Thus, each geometry is considered as a sub-geometry of another to which a certain basic figure is attached that must remain invariant. Projective geometry is a set of all invariants of set $S$ in relation to the group of projective transformations. Projective transformations are those applications that preserve the incidence relationship: the alignments. Affine geometry is the set of all invariants with respect to the affine group of transformations.

The affine group of transformations is a subgroup of the projective transformations. Parallelism is invariant to related transformations, etc. Other subgroups of the projective transformations are defined, and the equiform, Euclidean, pseudo-Euclidean, hyperbolic, and elliptic geometries are constructed [8]. In the following, we describe this process in more detail.

\section{Results - Systematization and classification of geometries by geometric transfor- mations}

The Erlangen program is a principle by which Klein gave the possibility of systematizing and classifying different areas of geometry.

We can observe any set $X$ called space, and the elements are the points of that space. The subsets of set $X$ are called figures. The transformation of space $X$ is called the bijective mapping $f$ of set $X$ to itself. Later we will treat projective geometrical transformations, but now we have to introduce specific concept of projective geometry, taken from [10]. We consider the concept of projectivity related to one-dimensional primitive forms, and later extend the concept to include all one-dimensional forms. We wish to extend the projectivity concept in another direction, so we will then be able to exhibit the relation between projective and Euclidean geometry in a more systematic manner. The extension we are now undertaking will be, of necessity, much less detailed than that in the book cited, for our purpose is merely to introduce certain ideas that are fundamental to the systematization of geometry by geometric transformations.

Extending the projective relationship to two-dimensional primitive forms is the basis of our investigation henceforth. The two-dimensional primitive forms are the field of points, the field of lines, the bundle of planes, and the bundle of lines. The last two are respectively the dual spaces of the first two, and because of this duality we restrict our statements for the most part to the field of points and the field of lines. Also, since two points determine a line, it is often convenient to consider, in conjunction with a field of points, its associated field of lines, and similarly, a field of lines with its associated field of points. Thus, the planar field is the totality of points and lines on one plane. Its dual space is called a bundle and is the totality of planes and lines on one point.

A planar field and a bundle are in perspective if they have no incident supports and if they are in a $(1,1)$ correspondence such that homologous elements, point and line or line and plane, are incident.

In the perspectivity, the elements common to the two perspective forms are self-corresponding elements. That is, every point on the intersecting line of the supports of two perspective fields of points is a self-corresponding point, and similarly, every plane on the 
line joining the centers of two perspective bundles of planes is a self-corresponding plane. Moreover, two perspective fields of points have the same projector from one point, the center of perspectivity, and two perspective bundles of planes have the same section by one plane, the plane of perspectivity.

To be more precise, we state the definition: A planar perspectivity is a $(1,1)$ correspondence between the elements of a plane which has like elements as homologues and leaves invariant every point on one line and every line on one point.

The support $o$ of the invariant point-row is called the axis of perspectivity, and the center 0 of the invariant flat pencil is called the center of perspectivity. A planar perspectivity whose axis and center are not incident is called a planar homology. If the axis and center are incident, the planar perspectivity is called a planar elation.

In an involutory or harmonic homology, homologous pairs are doubly corresponding. It is known as the following theorem: A planar perspectivity is uniquely determined if the center, axis, and one pair of homologous points are given, provided that the homologous points are not incident with the axis and are collinear with the center.

To prove this theorem, we shall show that when the center 0 , axis $o$, and homologous points $A, A^{\prime}$ are given, we can construct the homologue of any point $B$ of the plane.

Consider, first, the construction of the homologue of $B$ when $B$ is not on the line $O A$ $\left(=O A^{\prime}\right)$.

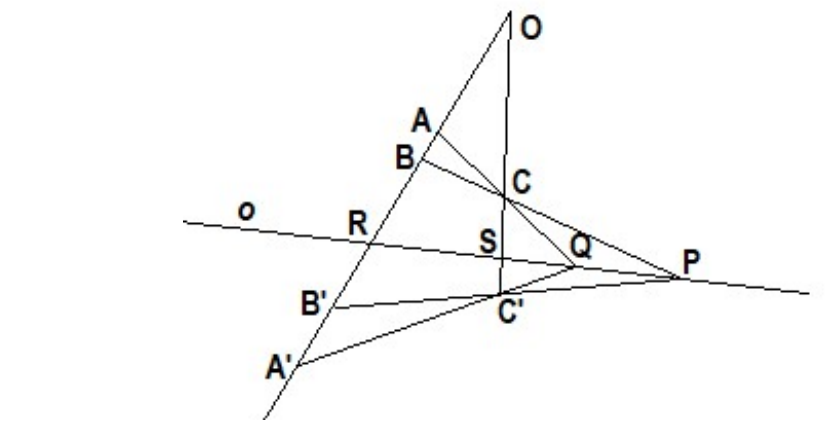

Figure 4. Construction of homologous points of planar perspectivity.

This homologue, call it $\mathrm{B}^{\prime}$, lies on $\mathrm{OB}$. Moreover, if $\mathrm{AB}$ cuts o at $\mathrm{R}$, we know that $\mathrm{R}$ is an invariant point, hence $A^{\prime} B^{\prime}$ must intersect $o$ at $R$. The homologue $B^{\prime}$ is then the intersection of $O B$ and $A^{\prime} R$, and as such is uniquely determined.

On the other hand, if $\mathrm{B}$ is a point on $\mathrm{OA}$, we construct any pair of homologous points $C, C^{\prime}$ not on OA. Then, by the same procedure but using the pair $C, C^{\prime}$ instead of $A, A^{\prime}$, we construct the homologue B'.

That the construction of B' is independent of the particular pair $C, C^{\prime}$ we chose follows immediately, in the case of a planar homology, as shown in Figure 4. For if $\mathrm{A}, \mathrm{A}^{\prime}$ meets o at $\mathrm{R}$ and if $\mathrm{C}, \mathrm{C}^{\prime}$ meets o at $\mathrm{S}$, we have: $A A^{\prime} O R \frac{Q_{1}}{\wedge} C C^{\prime} O S \frac{P}{\wedge} B B^{\prime} O R$, hence, $A A^{\prime} O R-B B^{\prime} O R$.

Now, this is a hyperbolic projectivity on $\mathrm{AA}^{\prime}$ and is completely determined by its double points 0 and $\mathrm{R}$ and the pair A, B (Patterson, 1958, p 77).

Consequently, $\mathrm{B}^{\prime}$ is unique, for it is the partner of $\mathrm{A}^{\prime}$ in that projectivity. If the planar perspectivity is a planar elation, the argument is much the same. Then, 0 coincides with $R$, the projectivity is parabolic, and $B^{\prime}$, the partner of $A^{\prime}$, is unique.

By the construction of a unique homologue of a given point, we have shown that the given data define not more than one planar perspectivity. To show that there is one such perspectivity, we construct a pair of space perspectivity's that satisfy the conditions of the theorem. This we shall leave as an exercise for the reader.

In general, there are two types of $(1,1)$ correspondences between the elements of two two-dimensional forms. If a $(1,1)$ correspondence between two forms is such that homologous elements are like elements and for every one-dimensional primitive form there is a corresponding one-dimensional primitive form, we call the correspondence a collineation. The planar perspectivity is a collineation between two superposed planar fields. 
In the other type of $(1,1)$ correspondence, homologous elements are not of the same kind, and this is called a correlation. We have in the pole-polar correspondence a particular kind of correlation between two superposed planar fields. For every point there is a corresponding line, its polar with respect to a given conic, and for every flat pencil there is a corresponding point-row. This correlation is a particular kind of correlation in two respects. First, it is projective, for it associates each flat pencil with a point-row projective, and second, it is involutory, for homologous elements are doubly corresponding.

We have indicated that all $(1,1)$ correspondences of the types mentioned above are not necessarily projective correspondences (Patterson, 1958). However, it is true in the domain of real projective geometry that all collineations and correlations are projective. Hence, since we shall restrict ourselves in what follows to real geometry, we can understand the term "collineation" when unqualified to mean projective collineation.

Consider now a set of transformations $S, T, R, \ldots$ such that the product of any two transformations of this set is a transformation of the set, and the inverse of each transformation is also contained in the set. Since the product of $S$ and S_1 must be a transformation of the set, the identity transformation belongs to the set. It is also evident that the transformations of this set are associative as to products, i.e., we can write:

$$
\mathrm{S}(\mathrm{TR})=(\mathrm{ST}) \mathrm{R}=\mathrm{STR}
$$

The transformations of a set such as we have described constitute a group of transformations. As an immediate example of a group of transformations, we mention the set of all projective transformations (including the identity) of a one-dimensional primitive form which transform that form into itself. It is evident from the definition of projectivity that the result of any two such projectivities is a projectivity of the set, and the inverse of any one projectivity is likewise a projectivity of the set.

Again, the set, including the identity transformation, of all collineations on a plane is a group. But, since the product of two planar correlations is a collineation, we see that the set of all correlations on a plane is not a group. On the other hand, the set of all collineations and all correlations on a plane is a group, called the general projective group of transformations on a plane.

\section{Affine geometry}

Among the projective collineations which transform a plane into itself, there is an important group of transformations which transform a given line of the plane into itself. This exceptional line of the plane is transformed into itself and is invariant, but the points of this line are not necessarily invariant points. So, the set of all projective collineations which leave invariant a fixed line of the plane is a group of transformations called the affine group of the plane.

Those properties of figures which remain invariant under this group of transformations but do not under the more general group of collineations are called affine properties, and the geometry which studies such properties is called affine geometry.

The affine group is contained in the collineation group, so is a subgroup of the collineation group. The invariant line of the affine group of transformations may be any line of the projective plane and is called the ideal line. Points on this exceptional line are ideal points, and all other points are ordinary points; all other lines are ordinary lines, and two ordinary lines which meet at an ideal point are parallel lines. The ordinary points and lines constitute the Euclidean plane. We observe that in a systematic way, the Euclidean plane is contained in the projective plane. Any figure or diagram which we may draw to illustrate ideas connected with the Euclidean plane as defined above will have the same appearance that one traditionally associates with such a figure or diagram only if the ideal line (the exceptional line of the plane) is considered to be identical with the line called by that name. It should be emphasized, however, that the ideal line of an affine transformation may be any line of the projective plane, but under such an interpretation, we must put aside tradition when viewing a figure or diagram.

Theorem 1 . There is a unique affine transformation which transforms the ordinary points $A, B, C$ into the ordinary points $A^{\prime}, B^{\prime}, C^{\prime}$, respectively. 
That this is a consequence of the fundamental theorem for determining the projective collineation between two planes is at once evident if we will replace the "points" of that theorem by "lines". Then it is seen that a collineation is uniquely determined when four pairs of homologous elements are given, and the four pairs of homologous elements here are the three pairs of sides of the two triangles, $A B C$ and $A^{\prime} B^{\prime} C^{\prime}$, and the ideal line, which corresponds to itself.

Theorem 2. Parallel lines are transformed into parallel lines by an affine transformation.

This follows, of course, from the fact that ideal points are transformed into ideal points.

Theorem 3. The ratio of division of a segment $A B$ by a point $C$ remains invariant under an affine transformation.

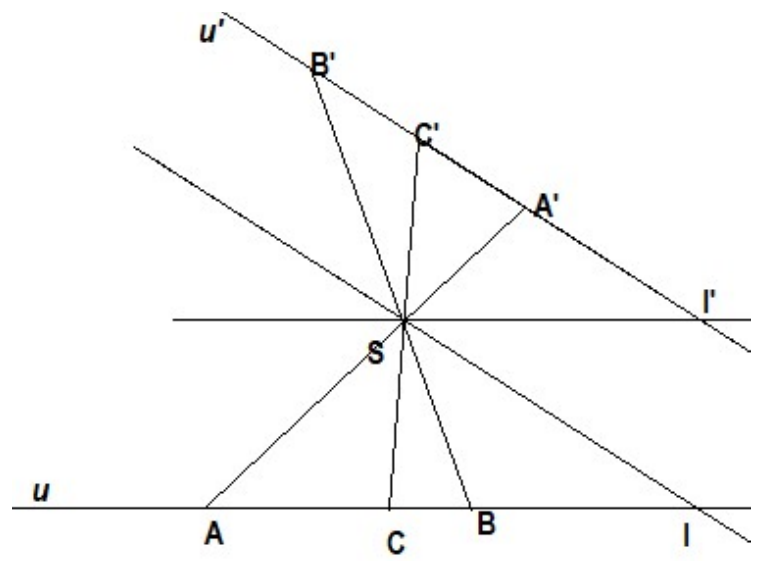

Figure 5. Invariant under affine transformation.

Line $l$ containing $A, B, C$ is transformed into line $l^{\prime}$ containing $A^{\prime}, B^{\prime}, C^{\prime}$. Moreover, if $I, I^{\prime}$ are respectively the intersections of $l, l^{\prime}$ with the ideal line, we have

$(A B, C I)=\left(\mathrm{A}^{\prime} \mathrm{B}^{\prime}, \mathrm{C}^{\prime} \mathrm{I}^{\prime}\right)$; that is, $\frac{A C}{C B}=\frac{A^{\prime} C^{\prime}}{C^{\prime} B^{\prime}}=$ a constant.

\section{Homothetic geometry}

In the homothetic group, any relation that has the ideal line as its axis is called a translation. If $l$ is an ordinary line through the center of the translation, the translation is said to be parallel to $l$.

From the general theory of elation, we know that the set of all translations is a group, and this group is a subgroup of the affine group. Since an elation is uniquely determined when its center, axis, and one pair of homologous points are known, it follows that there is one and only one translation sending a given point $A$ into a given point $A^{\prime}$. The center of this translation is, of course, the ideal point of line $A A^{\prime}$. A translation transforms every line not on its center into a parallel line and leaves invariant every line passing through its center.

Any homology that has an ideal line as its axis is called a dilation or a stretch. If 0 is the center of the stretch, the stretch is said to be about 0 . From the considerations, the set of all stretches on a plane is not a group; the product of two stretches is either a stretch or a translation. By definition, the set of all translations and all stretches on a plane is a group of transformations called the homothetic group of the plane. 


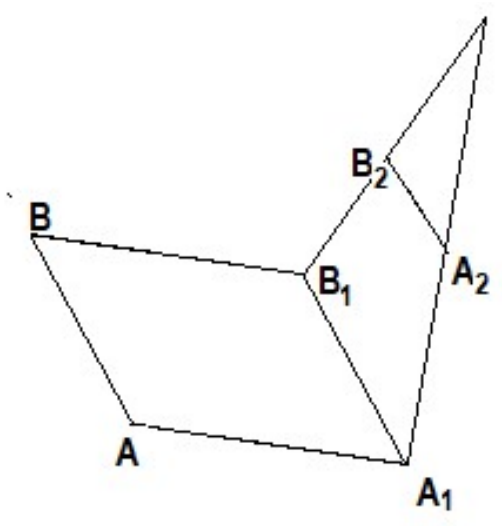

Figure 6. Translations and stretches.

A homothetic group is a subgroup of the affine, and a property invariant under the former but not the latter is called a homothetic property. The corresponding geometry is called homothetic geometry. Homothetic figures are perhaps more familiar as figures which are similar and similarly placed.

The transformations of a homothetic group are translations and stretches. Let $\mathrm{T}$ be a translation with center $\mathrm{O} 1$, and $\mathrm{S}$ a stretch with center $\mathrm{O} 2$. It is evident from Figure 6 that if $\mathrm{T}(\mathrm{A})=\mathrm{A} 1, \mathrm{~S}(\mathrm{~A})=\mathrm{A} 2$, then $\mathrm{T}(\mathrm{B})=\mathrm{B} 1, \mathrm{~S}(\mathrm{~B} 1)=\mathrm{B} 2$, and $\mathrm{T}(\mathrm{AB})=\mathrm{A} 1 \mathrm{~B} 1, \mathrm{~S}(\mathrm{~A} 1 \mathrm{~B} 1)=\mathrm{A} 2 \mathrm{~B} 2$, and $\mathrm{ST}(\mathrm{AB})=\mathrm{A} 2 \mathrm{~B} 2$ (Figure 6). Because $\mathrm{ABB} 1 \mathrm{~A} 1$ is a parallelogram and the segments $\mathrm{AB}$ and $\mathrm{A} 1 \mathrm{~B} 2$ are equal, under stretch $\mathrm{S}$, segments $\mathrm{A} 1 \mathrm{~B} 1$ and $\mathrm{A} 2 \mathrm{~B} 2$ are parallel, and the ratio of their lengths is a constant $\mu$ for transformation $S$. The constant $\mu$ is called the stretch factor of the transformation. These results can be stated as a theorem: Under a homothetic transformation, homologous segments are parallel, and the ratio of their lengths is constant.

\section{Similarity geometry}

An affine transformation leaves the ideal line invariant, but the points on that line are not invariant points. A homothetic transformation, on the other hand, leaves the ideal line and every ideal point invariant. We consider another subgroup of the affine group. For this purpose, we choose a fixed, but nevertheless arbitrary, elliptical involution on the ideal line. In what follows, we call it the absolute or orthogonal involution I, and two ordinary lines whose ideal points are homologues in this involution are said to be perpendicular lines.

The orthogonal involution, as defined here, may be any elliptical involution on the ideal line. Now we can provide a definition: The set of all projective collineations which leave invariant the orthogonal involution is a group of transformations called the similarity group of the plane. A property invariant under this group but not under the more general affine group is said to be a similarity property, and the corresponding geometry is called similarity geometry. Similarity geometry is, in fact, a part of Euclidian geometry in the larger sense, i.e., the Euclidian geometry which includes the theory of similar figures as well as the theory of congruent figures.

Consequently, homologous points of I are transformed into homologous points of I. In this we have a theorem: Perpendicular lines are transformed into perpendicular lines and parallel lines are transformed into parallel lines by a similarity transformation. It is evident from this those circles are transformed into circles by a similarity transformation.

\section{Metric geometry}

Now, we consider an involutory or harmonic homology which has center 0 , axis $\mathrm{o}$, and distinct points $\mathrm{A}, \mathrm{A}^{\prime}$ as homologues. Since $\mathrm{A}$ is transformed into $\mathrm{A}^{\prime}$, and since the pair $\mathrm{A}, \mathrm{A}^{\prime}$ is doubly corresponding, it follows that $\mathrm{A}^{\prime}$ is transformed into A. Therefore, if $\mathrm{AA}^{\prime}$ intersects the axis o at $\mathrm{B}$, we have $A A^{\prime} O R-A_{\wedge}^{\prime} A O R$ and $\left(\mathrm{AA}^{\prime}, 0 \mathrm{R}\right)=\left(\mathrm{A}^{\prime} \mathrm{A}, 0 \mathrm{R}\right)$. 
Also, $\left(\mathrm{AA}^{\prime}, \mathrm{OR}\right)=\frac{1}{\left(A^{\prime} A, O R\right)^{\prime}}$ and consequently, $\left(\mathrm{AA}^{\prime}, \mathrm{OR}\right)= \pm 1$.

The positive sign is discarded, for otherwise $A$ and $\mathrm{A}^{\prime}$ would not be distinct points. Hence, using the negative sign, we conclude that the pair A, A' harmonically separates the pair $0, R$. This proves the theorem: In an involutory or harmonic homology, pairs of homologous points are harmonically separated by the center of the homology and the intersection of their join with the axis of homology. In this way, we can define orthogonal line reflection as any harmonic homology whose center is an ideal point and whose axis intersects the ideal line at the point homologous to the center in the orthogonal involution. From this definition, it is evident that any pair of homologous ordinary points $\mathrm{A}, \mathrm{A}^{\prime}$ in an orthogonal line reflection $\mathrm{L}$ determines a segment $\mathrm{AA}^{\prime}$ which is perpendicular to and bisected by the axis of $\mathrm{L}$. If under $\mathrm{L}$ the ideal points I, I' are homologous, if $\mathrm{P}$ is the ideal point on the axis of $\mathrm{L}$, and if 0 is the center of $\mathrm{L}$, we have the harmonic relation $\mathrm{H}\left(\mathrm{I} \mathrm{I}^{\prime}, 0 \mathrm{P}\right)$. Also, under an orthogonal line reflection, the homologous segments $A B$ and $A^{\prime} B^{\prime}$ are equal in length, and homologous angles $A B C$ and $A^{\prime} B^{\prime} C^{\prime}$ are equal in magnitude but opposite in sign. That is, the distance between two points and the magnitude of the angle between two lines are invariant under such a transformation. Finally, we can state that the product of an even number of orthogonal line reflections is called a displacement or a rigid motion. The set of all rigid motions is a group of transformations called the metric group of the plane, which is a subgroup of the similarity group. Figures which are homologous under a transformation of the metric group are said to be congruent, a property invariant under this group but not under the similarity group is called a metric property, and the geometry which studies such properties is called metric geometry.

The distance between two points and the magnitude and sign of the angle between two lines remain invariant under a metric transformation. It is important to note that, for example, a circle is transformed into a circle by a rigid motion, but by an affine transformation it may be transformed into an ellipse; and similarly, foci and focal properties are invariant under metric but not affine transformations.

In the group of rigid motions, we have transformations which leave invariant one ordinary point. Hence, by the definition, the set of all rigid motions which leave invariant one ordinary point 0 is a group of transformations called the group of rotations about 0 . In particular, the product of two orthogonal line reflections whose axes intersect at an ordinary point 0 is a rotation about 0 . It can be shown that any displacement or rigid motion is the result of a rotation about a given point and a translation.

Thus, the metric group, the group of rigid motions, is exhibited as a special case of the general projective group. In doing so, we outline, in brief, the fundamental characteristics of other subgroups of the general projective group, but it should not be supposed that the groups and associated mentioned geometries exhaust the possibilities. There are others, but the all-inclusive nature of projective geometry and the significance of Cayley's remark, in his Sixth Memoir on Quantics, indicate that projective geometry is all geometry (Mumford et al., 2002).

\section{Möbius transformations}

To determine the group of transformations from $\mathrm{H}$ that map hyperbolic directions into hyperbolic directions, we need to expand our knowledge of hyperbolic directions. We will unite two seemingly different types of directions. Let us remember that there are directions contained in the Euclidean directions and in the Euclidean circles. We will notice that Euclidean circles can be obtained from Euclidean directions by adding one point.

Let $\mathrm{S} 1$ be a unit circle in $\mathrm{C}$, considering the function $\xi: \mathrm{S} 1 \backslash\{\mathrm{i}\} \rightarrow \mathrm{R}$ defined as follows: Let $\mathrm{z}$ be a point in $\mathrm{S} 1 \backslash\{\mathrm{i}\}$, and let $\mathrm{Kz}$ be a Euclidean line passing through points $\mathrm{i}$ and $\mathrm{z}$. The function is determined by $\xi(\mathrm{z})=\mathrm{R} \cap \mathrm{Kz}$. This function is well defined, because the intersection of $\mathrm{R}$ and $\mathrm{Kz}$ is a unique point and $\operatorname{Im}(\mathrm{z}) \neq 1$. Function $\xi$ is called a stereographic projection. 


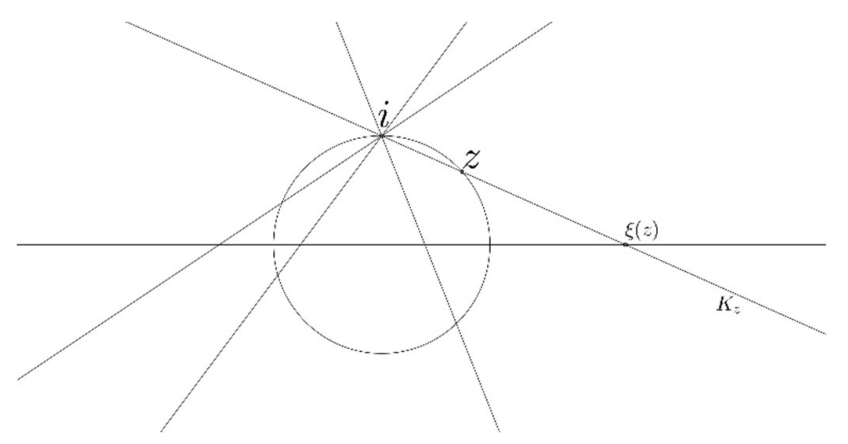

Figure 7. Stereographic projection.

Note that function $\xi$ is a bijective map of S1 without a single point to the real axis. We can think about the construction of a Euclidean circle starting from the Euclidean direction and adding one point. By adding one point to $\mathrm{C}$, we obtain a construction which in complex analysis is called a Riemann sphere, $\mathrm{Cr}$.

A Riemann sphere is the union of $\mathrm{Cr}=\mathrm{C} \cup\{\infty\}$ of the complex plane $\mathrm{C}$ and a point not contained in $\mathrm{C}$. We denote this point by $\infty$. We study the basic properties of $\mathrm{C}$, which means that the subset of $C r$ is open. Set $X$ is open in $C$ if for each $z \in X$ there exists $\varepsilon>0$ such that $U \varepsilon(\mathrm{z}) \subset X$, where $U \varepsilon(\mathrm{z})=\{\mathrm{w} \in \mathrm{C}|| \mathrm{w}-\mathrm{z} \mid<\varepsilon\}$ is an open Euclidean disk of radius $\varepsilon$ with the center at $z$. The set $X \subseteq C$ is closed if its complement is in open $C$.

Definition: Let $a, b, c, d \in C$ satisfy the condition ad - be $\neq 0$. A Mobius transformation is a mapping of $\mathrm{T}: \mathrm{C} \rightarrow \mathrm{C}$, determined as follows:

$$
\mathrm{T}(\mathrm{z})=\left\{\begin{aligned}
\frac{a z+b}{c z+d} ; & \text { where } z \neq-\frac{d}{c}, \infty \\
\infty ; \quad \text { where } z & =-\frac{d}{c} \\
\frac{a}{c} ; & \text { where } z=\infty
\end{aligned}\right.
$$

Example: Let us look at special cases.

If $\mathrm{a}=\mathrm{d}=1$ and $\mathrm{b}=\mathrm{c}=0$, then $\mathrm{T}(\mathrm{z})=\mathrm{z}$ is an identical transformation. We call other Mobius transformations nontrivial.

If $\mathrm{c}=0$, then $\mathrm{T}(\mathrm{z})=\frac{a}{d}+\frac{b}{d}$. This group consists of affine transformations written with the general form $\mathrm{T}(\mathrm{z})=\mathrm{A} z+\mathrm{B}$, where $\mathrm{A}, \mathrm{B}$ E C.

If $\mathrm{a}=\mathrm{d}=1$ and $\mathrm{c}=0$, then $\mathrm{T}(\mathrm{z})=\mathrm{z}+\mathrm{b}$ is the displacement.

If $b=c=0$ and $d=1$, then $T(z)=a z$ is an extension.

If $\mathrm{a}=\mathrm{d}=0$ and $\mathrm{b}=\mathrm{c}=1$, then $\mathrm{T}(\mathrm{z})=\frac{1}{\mathrm{z}}$ is the inversion.

If $\mathrm{AT}=\left(\begin{array}{ll}a & b \\ c & d\end{array}\right)$ is the matrix of Möbius transformation T, this assignment of the Möbius transformation also has nice properties, as follows.

First, $\mathrm{T}(\mathrm{z})$ is a (bijective) transformation if and only if $\operatorname{det} \mathrm{AT} \neq 0$; that is, if and only if AT is invertible.

Second, in this case, the inverse of the Möbius transformation corresponds to the inverse of the matrix, which corresponds to matrix $A_{T}^{-1}$, which has the associated Möbius transformation $\mathrm{T}-1$.

Then, the composition of Möbius transformations corresponds to matrix multiplication, that is, $\mathrm{AT} \circ \mathrm{BT}=\mathrm{ABT}$.

These three properties are summarized as the set of transformations $\mathrm{T}(\mathrm{z})$ is a homomorphism of groups SL2 (C).

Before we get acquainted with the classification of Möbius transformations, we need some new concepts on which it is based.

Definition: Let $\mathrm{M}=\left(\begin{array}{ll}a & b \\ c & d\end{array}\right) \in \mathrm{SL2}(\mathrm{C})$ be a matrix adapted to Möbius transformation $\mathrm{T}$. The sequence of Möbius transformation $\mathrm{T}$ is the sum of the coefficients $\mathrm{a}+\mathrm{d}$. We note that $\operatorname{TrT}$. 
Note 1: The trace of the Möbius transformation thus coincides with the trace of the transformation belonging to matrix $\mathrm{M}$, which is the sum of the elements on its principal diagonal.

Note 2: With the help of TrT, we can transform the formula for finding fixed points into the following form: $\mathrm{z} 1,2=\frac{a-d \pm \sqrt{(d-a)^{2}+4 b c}}{2 c}$, and taking into account ad $-\mathrm{be}=1$ for the expression under the root, we get $(d-a) 2+4 b c=(a+d) 2-4 a d+4 b c=(a+d) 2-4(a d-b c)$ $=(\mathrm{a}+\mathrm{d}) 2-4=\operatorname{TrT}$.

We can now introduce a classification of Möbius transformations. The criteria on which it is based are the value of $\operatorname{TrT}$ and the number of fixed points.

\section{Loxodromic transformations}

These are Möbius transformations that have two fixed points. They are characterized by the requirements that $\operatorname{Tr} T \in C \backslash[-2,2]$ and they are conjugate to the extension $\mathrm{T}(\mathrm{z})=\mathrm{kz}$, where $|\mathrm{k}|>1$.

A visualization of such a transformation of a complex plane reveals double spirals (motion originates from one fixed point and disappears into another), as shown in Figure 8.

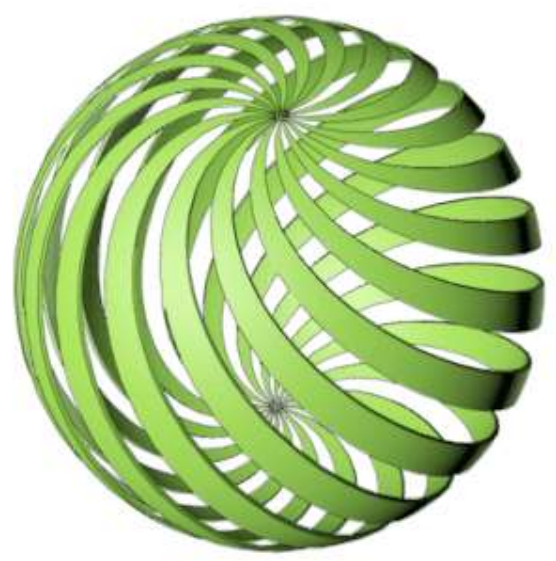

Figure 8. Double spherical spiral (M.C. Escher).

\section{Hyperbolic transformations}

These are Möbius transformations that also have two fixed points. Their characteristics are that the trace of the transformation is the real number $\operatorname{Tr} T \in C \backslash[-2,2]$ and they are conjugate to the stretch $\mathrm{T}(\mathrm{z})=\mathrm{kz}$, where $\mathrm{k} \in \mathrm{R}$ and $\mathrm{k}>1$.

What happens to the points of a complex plane in a hyperbolic transformation? A hyperbolic transformation can be understood as a subset of loxodromic transformations. In a hyperbolic Mobius transformation, the points do not move in spirals, but in circles through one fixed point to another.

Elliptical transformations: These are Möbius transformations that also have two fixed points. They are characterized by the requirement that TrT is a real number between -2 and 2 , and they are conjugated by a rotation $T(z)=k z$, where $|k|=1$.

To visualize an elliptic transformation, imagine two fixed points around which the other points of the plane move in circles.

Parabolic transformations: Obviously we also need to have a group of Möbius transformations that have only one fixed point. These are parabolic transformations. They are characterized by the requirement that $\operatorname{TrT}= \pm 2$, and they are conjugated by the displacement $\mathrm{T}(\mathrm{z})=\mathrm{z}+\mathrm{a}$.

In a parabolic transformation, the fixed point is a single point from which the orbits of the points emanate and to which they return, in a circular trajectory.

The coefficient $\mathrm{k}$ is also called the multiplier of the Möbius transformation. There is also a Möbius transformation that has more than two fixed points. There is a trivial 
Möbius transformation, $\mathrm{T}(\mathrm{z})=\mathrm{z}$, which cannot be placed in any of the groups mentioned. Each point of an identical transformation is its fixed point.

\section{Conclusion}

This description of the classification of geometries based on geometric transformations is made on the basis of the concept of the invariant of a determined group of transformations. It is well known that we will be able to reach the same classification of geometries through deductive theory (axioms and basic concepts given before, continuing the construction of the derived concepts and the series of statements - theorems).

Now, the questions are raised which will be of interest to study the way of constructing geometry based mainly on geometric transformations as a way of geometric thinking.

Author Contributions: "Conceptualization, J.G. and X.T.; methodology, J.G and Y.V.; software, Y.V.; validation, J.G., X.T. and Y.V.; formal analysis, J.G.; investigation, J.G. and X.T.; resources, J.G.; writing - original draft preparation, J.G.; writing - review and editing, X.T.; visualization, Y.V.; supervision, J.G.; funding acquisition, J.G. All authors have read and agreed to the published version of the manuscript."

Acknowledgments: Acknowledge th support given by DMNE of University of Barcelona for permanently support during the process of preparing this article.

Conflicts of Interest: "The authors declare no conflict of interest." "The funders had no role in the design of the study; in the collection, analyses, or interpretation of data; in the writing of the manuscript, or in the decision to publish the results".

\section{References}

1. Weyl, H. Symmetry. Princeton U.P., Parcialmente reimpreso en El mundo de las matemáticas, Vol. 4, Grijalbo, 1983.

2. Forgarty, J.; Mummford, D. Geometric Invariant Theory. Springer: Verlag, 1982, ISBN 978-3-540-56963-3.

3. Mumford, D.; Series, C.; Wright, D. Indra's pearls: The vision of Felix Klein, Cambridge University Press: Cambridge, 2002. ISBN 0-521-35253-3.

4. Zisserman, A.; Mundy, J. (Eds.) Proceedings of the DARPA - ESPRIT workshop on Applications of Invariants in Computer Vision, Reykjavik, Iceland, March 1991.

5. Wolfson, H. J. Model-based object recognition by geometric hashing. In Proceedings of the 1st European Conference on Computer Vision, Antibes, France, April 1990; Springer: Verlag; pp. 526-536.

6. Kapur, D., Mundy J.L. Fitting 6ne invariant conics to curves. In Proceeding of the DARPA-ESPRIT workshop on Applications of Invariants in Computer Vision, Reykjavik, Iceland, March 1991; pp. 209-233. https://hal.inria.fr/inria-00590013

7. Gros, P.; Quan, L. Projective Invariants for Vision. Technical Report RT 90 IMAG - 15, LIFIA, Lifia-Irimag, Grenoble, France, December 1992.

8. $\quad$ Patterson, B.C. Projective Geometry, John Wiley \& Sons, Inc: New York, 1937.

9. Lodge, O. Johan Kepler. En Sigma El mundo de las Matemáticas, Grijalbo: Barcelona, 1983 Volume 1, pp. 146-161.

10. Thaqi, X. Aprender a enseñar transformaciones geométricas en Primaria desde una perspectiva cultural. Unpublished phD Thesis. Universitat de Barcelona. 2009.

11. Font, V. Epistemología y didáctica de las matemáticas. En F. Ugarte (ed.) Reportes de investigación 21, serie C, II Coloquio internacional sobre Enseñanza de las Matemáticas. Lima. Perú PUCP (pp 1-48), 2007. 